\title{
Graded contribution of hippocampus to multifeature binding across temporal delay

\author{
Lei Zhu ${ }^{\mathrm{a}, \mathrm{d}}$, Xiuyan Guo a,b,d ${ }^{\mathrm{a}}$ Li Zheng ${ }^{\mathrm{b}}$, Jianqi Li ${ }^{\mathrm{c}, \mathrm{d}}$, Mengchao Pei ${ }^{\mathrm{c}, \mathrm{d}}$, \\ Zoltan Dienes ${ }^{\mathrm{e}}$ and Zhiliang Yang ${ }^{\mathrm{b}}$
}

\begin{abstract}
It is well accepted that all the elements of an episode are combined together by the hippocampus to form an episodic representation. In this study, we further investigated whether the activation pattern of the hippocampus during multifeatural episodic encoding with temporal discontinuities varies with recollective detail in a graded way. Specifically, in the present functional magnetic resonance imaging study, we manipulated two associative features, color and size, and presented the features and the item sequentially. Right hippocampal activation increased with the number of features successfully bound to the item, supporting a graded role of hippocampal activation in bridging temporal discontinuities for integrating multiple episodic features.
\end{abstract}

\author{
NeuroReport 21:902-906 (c) 2010 Wolters Kluwer Health | \\ Lippincott Williams \& Wilkins. \\ NeuroReport 2010, 21:902-906
}

Keywords: episodic memory, graded contributions, hippocampus

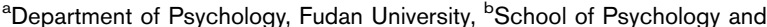
Cognitive Science, ' Department of Physics, dShanghai Key Laboratory of Magnetic Resonance, East China Normal University, Shanghai, China and ${ }^{\mathrm{e}}$ School of Psychology, University of Sussex, Brighton, UK

Correspondence to Xiuyan Guo, PhD, School of Psychology and Cognitive Science, East China Normal University, Shanghai, SH 200062, China Tel: +862162233484; fax: + 862167644201 ; e-mail: xyguo@psy.ecnu.edu.cn

Received 22 June 2010 accepted 4 July 2010

hippocampus during multifeatural episodic encoding would offer key insights into the neural basis of the episodic memory.

In considering the possible hippocampal engagement during multifeatural episodic encoding, how the episodic features are manipulated is likely to be important. It is widely accepted that the hippocampus is critically engaged in bridging temporal discontinuities for integrating sequential items together [13]. The hippocampus was found to be involved in feature binding when there was temporal separation between the item and the related features $[9,10,14]$. However, to our knowledge, none of the earlier studies on multifeatural episodic encoding $[8,11,12]$ presented the item and its related features sequentially. Therefore, in our study, the related features and the items appeared sequentially with a $0.5 \mathrm{~s}$ interval.

Another consideration is which features are embedded in the episode. One dichotomy is to distinguish associative from organizational features [15]. Associative features refer to those features, which are directly connected with the item and may be encoded concurrently with the item, such as color, shape, and size. Organizational features refer to features, which are extrinsic to the item and require more effort to encode, such as spatial location and temporal order. It has been argued that the hippocampus is selectively engaged during episodic encoding for associative features, but not organizational features [15]. However, except for the associative features (i.e. color), Uncapher et al. [8] and Staresina and Davachi [12] also manipulated the organizational features (i.e. location 
or task), which might less involve hippocampus [15] and more rely on cortical regions other than hippocampus, such as frontal cortex in Uncapher et al. [8], during feature binding.

Altogether, in the present event-related fMRI study, we manipulated two associative features, color and size, independently in the same manner, presented the features and the item sequentially, and tested how hippocampus activity would be modulated by the number of features subsequently recollected under temporal discontinuities.

\section{Methods}

\section{Participants}

Fifteen right-handed volunteers from the university community with normal or corrected-to-normal vision (six males and nine females, aged from 18-28 years) participated in this study. All the participants were paid $100 \mathrm{RMB}$ for their participation. Written informed consent was obtained in a manner approved by the Ethics Committee of the Shanghai Psychological Society.

\section{Materials}

Four hundred and fifty black and white pictures of common objects were randomly selected from International Picture-Naming Project by Center for Research in Learning at University of California, San Diego picture database as materials, 300 of which were randomly selected to serve as learning items and 150 of which served as lures during the memory test.

\section{Procedure}

During the scanned encoding phase, 300 learning items were presented sequentially. For each encoding trial, a $0.5 \mathrm{~s}$ frame cue varying in size and color was presented at first, followed by a $0.5 \mathrm{~s}$ blank interval (blank screen) and a $3 \mathrm{~s}$ black and white object. Participants were instructed to form a mental image of each object according to the frame color and cue size, and indicated whether they could successfully form such a mental image. For the color, participants were instructed to imagine the object in the frame color. For the size, participants were instructed to imagine the object to be as big as a $20 \mathrm{~m}^{2}$ room if the frame was big and as small as a shoe box if the frame was small. Responses were given through a magnetcompatible button box below participants' left hand. The encoding trials were intermixed with $4 \mathrm{~s}$ null trials (75 trials in all), during which a black fixation cross was presented. All the trials were presented with jittered interstimulus intervals (a fixation cross) from 0-1 s.

About 15 min after the encoding phase, participants were given a surprise self-paced memory test outside the scanner. Four hundred and fifty objects were presented sequentially in a random order. Participants were instructed to indicate whether the object was old (presented during the encoding phase) or new (not presented during the encoding phase). If they responded as 'old', they were then required to indicate the related color and size of their mental image of the object.

\section{Functional magnetic resonance imaging}

Imaging was carried out on a $3 \mathrm{~T}$ Siemens scanner at the Functional MRI Lab (East China Normal University, Shanghai). Functional imaging was acquired using a gradient echo-planar imaging sequence [repetition time $(\mathrm{TR})=2200 \mathrm{~ms}$, echo time $=30 \mathrm{~ms}$, field of view $=$ $220 \mathrm{~mm}$, matrix size $=64 * 64$ ]. Thirty-five slices perpendicular to the anterior commissure-posterior commissure line (slice thickness $=3 \mathrm{~mm}$, gap $=0.3 \mathrm{~mm}$ ) were acquired and covered the whole brain. The first five TRs acquired were discarded to allow for T1 equilibration. Before the two functional runs, a high-resolution structural image was acquired using a T1-weighted, multiplanar reconstruction sequence $(\mathrm{TR}=1900 \mathrm{~ms}$, echo time $=3.42 \mathrm{~ms}, 192$ slices, slice thickness $=1 \mathrm{~mm}$, field of view $=256 \mathrm{~mm}$, matrix size $=256 \times 256$ ).

\section{Functional magnetic resonance imaging data analysis}

Data preprocessing and statistical analyses were performed with Statistical Parametric Mapping (SPM5, Wellcome Department of Cognitive Neurology, London, UK). During data preprocessing, all volumes were corrected for differences in slice acquisition timing and realigned spatially to the first volume of the first time series. None of participants moved more than $3 \mathrm{~mm}$ in any direction during the encoding phase. Then, the resulting images which were resampled to $2 \times 2 \times 2 \mathrm{~mm}$ voxel size were spatially normalized to a standard echo-planar imaging template based on the Montreal Neurological Institute reference brain and smoothed with an $8 \mathrm{~mm}$ full-width half-maximum isotropic Gaussian kernel.

Statistical analyses were performed using the general linear model implemented in SPM5. Encoding trials were classified into four conditions according to participants' performance at subsequent memory tests: (i) learning items later judged to be new (MISS), (ii) learning items later recognized without correct judgment of both the color and the size (Item Only or IO), (iii) learning items later recognized with only one associative feature (color or size) being correctly judged (ONE), (iv) learning items later recognized with both associative features being correctly judged (TWO). Each encoding trial was modeled using a canonical hemodynamic response function and its temporal derivative according to four memory conditions. All the encoding trials were modeled as $4 \mathrm{~s}$ long events from the onset time of the frame cue. Six regressors modeling movement-related variance and one modeling the overall mean during the whole encoding phase were also employed in the design matrix. Parameter estimates ( $\beta$ estimates in the figures) for each regressor of interest at the single subject level were submitted to the second-level 
group analysis. Each participant's $\beta$ estimates for the four memory conditions were entered into a flexible factorial analysis of variance (ANOVA).

Importantly, additional parametric analyses, an efficient statistical procedure to reveal voxels that show a particular pattern of activation throughout several conditions [16], was conducted at the individual-subject fixedeffects level to assess how hippocampus activity modulated by the amount of features subsequently recollected. Specifically, a parametric regressor was created to represent the different weighting for different memory conditions $(\mathrm{IO}=1, \mathrm{ONE}=2$, TWO $=3)$. The resulting subjectspecific estimates of the parametric regressor at each voxel were then entered into a second-level one sample $t$-tests treating participants as a random variable.

In the whole-brain analysis, the resulting statistical map was thresholded at a voxel-wise $P$ value less than 0.001 and a spatial-extent threshold of 10 contiguous voxels. For parametric analyses, as the purpose of this study was to test how hippocampus activity would be modulated by the number of features subsequently recollected, we focused our analyses on the hippocampus and a small volume correction (SVC) was applied to the hippocampus bilaterally. For this purpose, an anatomical SVC mask of hippocampus was created by anatomy toolbox [17]. Clusters resulted from parametric analyses exceeding the threshold of $P$ value less than 0.05 (family wise error corrected) after an SVC were considered reliable. Anatomic labeling of activations of clusters was performed with the anatomy toolbox. Peak voxels were reported in Montreal Neurological Institute coordinates.

\section{Results}

\section{Behavioral results}

In the memory test, $67 \%$ of the learning items were judged as old (hits) and $18 \%$ of the lures were judged as old (false alarms). There were 99 [standard deviation $(\mathrm{SD})=$ 29], $31(\mathrm{SD}=11), 85(\mathrm{SD}=16), 85(\mathrm{SD}=29)$ trials in the MISS, IO, ONE, and TWO memory conditions, respectively. The average accuracy of associative memory for color $[0.61 \quad(S D=0.09)]$ and that for size $[0.65$ $(\mathrm{SD}=0.10)]$ were not significantly different from each other $[t(14)=1.72, P>0.05]$. However, both the accuracy for color and size were significantly above the chance level of $50 \%$ [color: $t(14)=4.77, P<0.01$; size: $t(14)=5.71$, $P<0.01]$.

\section{Functional magnetic resonance imaging results}

\section{Additive effect of feature binding}

The parametric analyses revealed that the only hippocampal cluster showing additive effect of feature binding after an SVC (family wise error, $P<0.05$ ) was located in the right anterior hippocampus extended to entorhinal cortex (peak xyz: 16, $-10,-24$, significant $t=5.37$, Fig. 1a). Participants' $\beta$ estimates for three regressors
Fig. 1

(a)

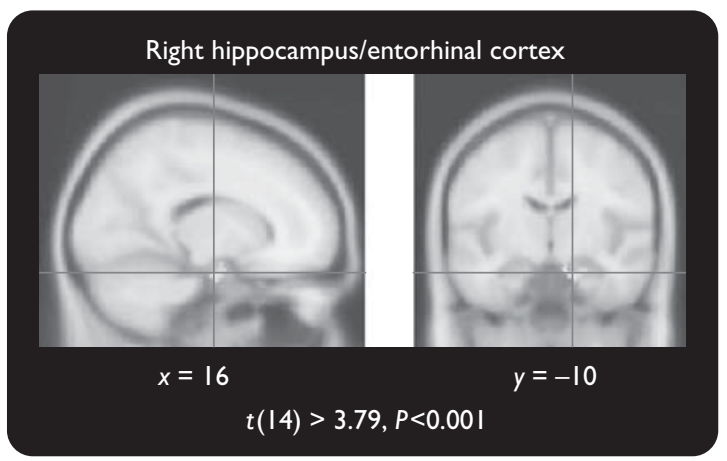

(b)

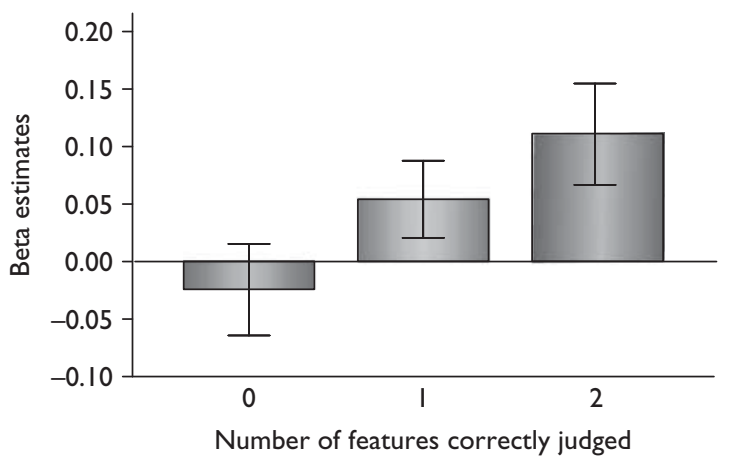

Additive effect of number of features binding in the right hippocampus according to parametric analyses. (a) Hippocampal cluster extended to entorhinal cortex resulting from parametric analyses after small volume correction (family wise error, $P<0.05$ ) showing additive effect of number of features binding. (b) Average $\beta$ estimates across right hippocampal cluster extended to entorhinal cortex in IO, ONE, TWO conditions. Hippocampal activation increased with the number of features successfully bound to the item. Error bars indicate standard error of the mean.

corresponding to IO, ONE, TWO conditions were derived across the right hippocampal cluster and are depicted in Fig. 1b, which indicated activation of the hippocampus increased with the number of features successfully bound to the item. The stepwise increments were confirmed by several $t$-tests. The activation of the right hippocampus cluster was significantly higher in ONE condition than in IO condition $[t(14)=3.03, P<0.01]$ and significantly higher in TWO condition than in ONE condition $[t(14)=$ 2.67, $P<0.05]$.

Next, on the basis that the parametric analysis can only reveal regions which bear a preset pattern of activation (e.g. linear), we questioned whether there existed other regions in the hippocampus except for the beforementioned regions whose activity did not follow a graded pattern. On the basis of the vital role of the hippocampus in feature binding, activity in such hippocampal regions was supposed to be greater in both ONE and TWO conditions than in IO condition. Hence, to search for such regions, we conducted a four memory conditions ANOVA (see fMRI Data Analysis) and applied a conjunction analysis [18] of two contrasts: TWO versus IO and 
ONE versus IO within this ANOVA model (individual contrast thresholded at $P<0.001$ to result in a conjunction threshold of $P<0.000001)$. Interestingly, the only cluster in the whole brain emerged from conjunction analysis overlapped partly with the right hippocampal cluster extended to entorhinal cortex reported above (peak $x y \%$ : $16,-10,-24$, significant $t=3.91$ ). Participants' $\beta$ estimates for three regressors corresponding to IO, ONE, TWO conditions were derived across the cluster, which indicated a stepwise increased activation in this cluster. Following $t$-tests showed the right hippocampal activation was significantly higher in ONE condition than in IO condition $[t(14)=3.27, P<0.01]$ and significantly higher in TWO condition than in ONE condition $[t(14)=2.59$, $P<0.05]$. Thus, it is confirmed that the hippocampus activity might be modulated by the number of features subsequently recollected.

\section{Item memory effect}

However, it was too early to draw conclusion here. One alternative explanation for the above result was that the graded contributions of the hippocampus is related to the amount of remembered information in general, but not specific to the successful binding between associative features and the item, which predicts greater hippocampal activity in IO condition than in MISS condition. To confirm the graded contributions of the hippocampus specific to feature binding, but not to the general item memory effect, we extracted $\beta$ estimates in IO and MISS conditions across the hippocampus cluster from parametric analysis. It was found that hippocampal activity in IO condition was the same as that in MISS condition $[t(14)=1.56, P>0.05]$, providing no evidence that the hippocampus subserves item memory. This null result may be because of a lack of sensitivity. Thus, to determine the size of effect, we might expect to obtain, we searched for regions that exhibited item memory effects by contrasting IO and MISS conditions. The only medial temporal lobe region surviving in this analysis is located in the right perirhinal cortex (xyz: 46, $-20,-28$, significant $t=4.47$ [19]). Further analysis on $\beta$ estimates extracted from the local maximum voxel revealed that activity in the right perirhinal cortex showed a significant item memory effect $[t(14)=3.22, P<0.01]$, which was crucially significantly different from that in the hippocampus $[\mathrm{F}(1,14)=13.23, P<0.01]$. It indicated that we can sensitively exclude an item effect in the hippocampus. In summary, the result implied that the graded contributions of the hippocampus were specific to feature binding essential for episodic memory formation.

\section{Discussion}

This study was designed to elucidate the specific activation pattern of the hippocampus during multifeatural episodic encoding. The finding that the right hippocampal activity increased with the number of features successfully bound to the item is consistent with previous fMRI studies showing activity in the hippocampus was greater during encoding of items for which the related features were subsequently remembered rather than forgotten $[5-10,12]$. We extend these results by showing an additive effect of number of features binding in the hippocampus ( $c f$. [12]). However, our research used two associative features each of equal performance difficulty, which might allow the hippocampus to be involved at the same extent for two features [15]. Furthermore, in line with brain lateralization perspective [20], we used pictures as materials and found hippocampal activation in the right hemisphere. Finally, most importantly, we presented the features and the item sequentially showing that binding multiple episodic features across the temporal delay mainly relied on the graded contributions of the hippocampus.

Moreover, the temporal separation between the item and the features is likely to be critical when understanding the functional dissociation between different regions in the medial temporal lobe. In this study, the graded contribution of hippocampus was not found to be related to item recognition, but to be limited to the binding between item and its related features, whereas activity in the right perirhinal cortex showed item memory effects. At first glance, this result is in line with the functional dissociation between different regions in the medial temporal lobe: the hippocampus is critical for later recovery of associated contextual features, whereas the perirhinal cortex is critical for later item recognition $[5,6,10]$.

However, there has been increasing evidence against functional dissociations in different regions of the medial temporal lobe. Specifically, the perirhinal cortex has been shown to be engaged in associative memory formation in several studies [11,12,21-23]. Upon closer examination, we found that all the above studies required participant to bind a coherent associative feature presented simultaneously with the target item. A relevant distinction might be whether or not binding occurs over an extended period of time. Binding processes of simultaneous visual features (e.g. a pen and its color 'blue' in the real world) may involve the late stages of the ventral visual stream at the parahippocampal gyrus, particularly perirhinal cortex ([24]; for review see Ref. [25]). In contrast, binding of temporally separated features may rely on the hippocampus. Consistent with this scenario, our study, together with several other studies $[9,10,14]$ found a hippocampal, but no perirhinal binding effect when there was temporal separation between the item and the related features. Further investigations are needed to verify the above hypothesis.

\section{Conclusion}

This study revealed that hippocampus produced a graded activation according to the number of (temporally separated) features bound together. 


\section{Acknowledgements}

The authors would like to thank Professor Daniel L. Schacter (Harvard University, Cambridge) for his comments on an earlier version of this manuscript. This study was carried out at East China Normal University (North Zhongshan Road 3663, Shanghai, SH 200062, China).

\section{References}

1 Tulving E. Episodic memory: from mind to brain. Ann Rev Psychol 2002; 53:1-25.

2 Diana RA, Yonelinas AP, Ranganath C. Imaging recollection and familiarity in the medial temporal lobe: a three-component model. Trends Cogn Sci 2007; 11:379-386.

3 Eichenbaum $\mathrm{H}$, Yonelinas $\mathrm{A}$, Ranganath $\mathrm{C}$. The medial temporal lobe and recognition memory. Ann Rev Neurosci 2007; 30:123-152.

4 Honey RC, Watt A, Good M. Hippocampal lesions disrupt an associativemismatch process. J Neurosci 1998; 18:2226-2230.

5 Davachi L, Mitchell JP, Wagner AD. Multiple routes to memory: distinct medial temporal lobe processes build item and source memories. Proc Nat Acad Sci U S A 2003; 100:2157-2162.

6 Ranganath C, Yonelinas AP, Cohen MX, Dy CJ, Tom SM, D'Esposito M. Dissociable correlates of recollection and familiarity within the medial temporal lobes. Neuropsychologia 2003; 42:2-13.

7 Kensinger EA, Schacter DL. Amygdala activity is associated with the successful encoding of item, but not source, information for positive and negative stimuli. J Neurosci 2006; 26:2564-2570.

8 Uncapher MR, Otten LJ, Rugg MD. Episodic encoding is more than the sum of its parts: an fMRI investigation of multifeatural contextual encoding. Neuron 2006; 52:547-556.

9 Staresina BP, Davachi L. Mind the gap: binding experiences across space and time in the human hippocampus. Neuron 2009; 63:267-276.

10 Qin S, Rijpkema M, Tendolkar I, Piekema C, Hermans EJ, Binder M, et al. Dissecting medial temporal lobe contributions to item and associative memory formation. Neuroimage 2009; 46:874-881.

11 Tendolkar I, Arnold J, Petersson KM, Weis S, Anke BD, van Eijndhoven P, et al. Probing the neural correlates of associative memory formation: a parametrically analyzed event-related functional MRI study. Brain Res 2007; 1142:159-168.

12 Staresina BP, Davachi L. Selective and shared contributions of the hippocampus and perirhinal cortex to episodic item and associative encoding. J Cogn Neurosci 2008; 20:1478-1489.

13 Wallenstein GV, Eichenbaum $\mathrm{H}$, Hasselmo ME. The hippocampus as an associator of discontiguous events. Trends Neurosci 1998; 21:317-323.

14 Qin S, Piekema C, Petersson KM, Han B, Luo J, Fernandez G. Probing the transformation of discontinuous associations into episodic memory: an event-related fMRI study. Neuroimage 2007; 38:212-222.

15 Moscovitch M. Memory and working-with-memory: a component process model based on modules and central systems. J Cogn Neurosci 1992; 4:257-267.

16 Buchel C, Holmes AP, Rees G, Friston KJ. Characterizing stimulusresponse functions using nonlinear regressors in parametric fMRI experiments. Neuroimage 1998; 8:140-148.

17 Amunts K, Kedo O, Kindler M, Pieperhoff $\mathrm{P}$, Mohlberg $\mathrm{H}$, Shah NJ, et al. Cytoarchitectonic mapping of the human amygdala, hippocampal region and entorhinal cortex: intersubject variability and probability maps. Anatomy Embryol 2005; 210:343-352.

18 Nichols T, Brett M, Andersson J, Wager T, Poline JB. Valid conjunction inference with the minimum statistic. Neuroimage 2005; 25:653-660.

19 Holdstock JS, Hocking J, Notley P, Devlin JT, Price CJ. Integrating visual and tactile perceptual information in the perirhinal cortex. Cerebral Cortex 2009 19:2993-3000.

20 Toga AW, Thompson PM. Mapping brain asymmetry. Nat Rev Neurosci 2003; 4:37-48.

21 Jackson O, Schacter DL. Encoding activity in anterior medial temporal lobe supports subsequent associative recognition. Neuroimage 2004; 21:456-462.

22 Kirwan CB, Stark CE. Medial temporal lobe activation during encoding and retrieval of novel face-name pairs. Hippocampus $2004 ; 14: 919-930$.

23 Staresina BP, Davachi L. Differential encoding mechanisms for subsequent associative recognition and free recall. $J$ Neurosci 2006; 26 : 9162-9172.

24 Taylor Kl, Moss HE, Stamatakis EA, Tyler LK. Binding crossmodal object features in perirhinal cortex. Proc Natl Acad Sci U S A 2006; 103:8239-8244.

25 Bussey TJ, Saksida LM, Murray EA. The perceptual-mnemonic/feature conjunction model of perirhinal cortex function. Q J Exp Psycho/ 2005; 58:269-282. 